University of Nebraska - Lincoln

DigitalCommons@University of Nebraska - Lincoln

2010

Structural, magnetic and magneto-transport properties of Ptalloyed $\mathrm{MnBi}$ thin films

\author{
Parashu Kharel \\ University of Nebraska-Lincoln, pkharel2@unl.edu \\ Ralph Skomski \\ University of Nebraska-Lincoln, rskomski2@unl.edu \\ Roger D. Kirby \\ University of Nebraska-Lincoln, rkirby1@unl.edu \\ David J. Sellmyer \\ University of Nebraska-Lincoln, dsellmyer@unl.edu
}

Follow this and additional works at: https://digitalcommons.unl.edu/physicsskomski

Part of the Physics Commons

Kharel, Parashu; Skomski, Ralph; Kirby, Roger D.; and Sellmyer, David J., "Structural, magnetic and magneto-transport properties of Pt-alloyed MnBi thin films" (2010). Ralph Skomski Publications. 61. https://digitalcommons.unl.edu/physicsskomski/61

This Article is brought to you for free and open access by the Research Papers in Physics and Astronomy at DigitalCommons@University of Nebraska - Lincoln. It has been accepted for inclusion in Ralph Skomski Publications by an authorized administrator of DigitalCommons@University of Nebraska - Lincoln. 


\title{
Structural, magnetic and magneto-transport properties of Pt-alloyed MnBi thin films
}

\author{
P. Kharel, ${ }^{\text {a) }}$ Ralph Skomski, R. D. Kirby, and D. J. Sellmyer \\ Department of Physics and Astronomy, Nebraska Center for Materials and Nanoscience, University of \\ Nebraska, Lincoln, Nebraska, 68588, USA
}

(Presented 21 January 2010; received 30 October 2009; accepted 25 December 2009; published online 3 May 2010)

The structural, magnetic and magneto-transport properties of highly c-axis oriented $\mathrm{Mn}_{55_{-}} \mathrm{Pt}_{x} \mathrm{Bi}_{45}$ $(x=0,1.5,3$, and 4.5$)$ thin films have been investigated. The coercivity of the Pt-alloyed thin films increases and the saturation magnetization decreases as the $\mathrm{Pt}$ concentration increases. The anisotropy field $H_{o}$ increases as a function of Pt concentration, too but the coercivity increases more rapidly than the anisotropy field. This indicates an enhanced domain-wall pinning, caused by increased interstitial disorder due to the occupancy of regular Mn sites by Pt. The same mechanism explains the reduced magnetization. All samples exhibit a large extraordinary Hall effect with anomalous Hall coefficient about an order of magnitude larger than the ordinary Hall coefficient. (C) 2010 American Institute of Physics. [doi:10.1063/1.3360204]

\section{INTRODUCTION}

There has been considerable interest in understanding the magnetic, optical, and magneto-transport properties of MnBi-based materials because of their substantial magnetooptical and permanent magnet properties. ${ }^{1} \mathrm{MnBi}$ exhibits interesting properties including an extraordinarily large Kerr rotation, ${ }^{2}$ a Curie temperature well above room temperature, ${ }^{3}$ a high coercivity with a rectangular hysteresis loop, ${ }^{4}$ and a large perpendicular anisotropy in thin films at room temperature. ${ }^{5}$

Pure MnBi adopts the hexagonal NiAs-type crystal structure at room temperature with lattice constants of $a$ $=4.29 \AA$ and $c=6.12 \AA \AA^{6}$ Around $628 \mathrm{~K}$, it undergoes coupled structural and magnetic phase transitions from a ferromagnetic low-temperature phase (LTP) to a paramagnetic high-temperature phase (HTP). ${ }^{4}$ If MnBi in the HTP is rapidly quenched, a ferromagnetic quenched HTP (QHTP) with a Curie temperature of about $440 \mathrm{~K}$ is obtained. ${ }^{7}$ QHTP is thermally unstable and transforms slowly back to LTP at room temperature with a time constant of about two years. ${ }^{8}$ In addition to the structural instability, MnBi in LTP shows an increasing coercivity as temperature increases reaching a maximum at $553 \mathrm{~K}^{4}$ These circumstances have led to several studies of the effect of dopants on the various properties of this material. ${ }^{9-14}$ Some of these experiments show that the structural, magnetic, and magneto-optical properties of $\mathrm{MnBi}$ can be controlled by doping it with a third element. The goal of this study is to understand and control the structural, magnetic, and magneto-transport properties of MnBi-based alloys by alloying with the heavy and noble element Pt.

\section{EXPERIMENTAL METHODS}

We have synthesized Pt-alloyed MnBi thin films having post-annealed stoichiometry of $\mathrm{Mn}_{55-x} \mathrm{Pt}_{x} \mathrm{Bi}_{45}$ ( $x=0,1.5,3$, and 4.5) by sequential evaporation of $\mathrm{Bi}, \mathrm{Mn}$, and $\mathrm{Pt}$ onto a glass substrate at room temperature using an AJA e-beam

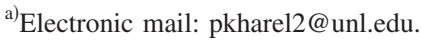

evaporation system. The base pressure of the evaporation chamber was $4 \times 10^{-9}$ Torr. Bi and Mn were evaporated from alumina crucibles and $\mathrm{Pt}$ was evaporated from a graphite crucible. The atomic ratios of the constituent elements in the $\mathrm{Mn}_{55-x} \mathrm{Pt}_{x} \mathrm{Bi}_{45}$ films were estimated from the respective layer thicknesses and were later confirmed using energy dispersive $\mathrm{x}$-ray (EDX) spectroscopy. The deposition layer thickness was controlled by a quartz crystal thickness monitor. All the samples were annealed in situ immediately after deposition at a temperature of $375^{\circ} \mathrm{C}$ for one hour. The thicknesses of the postannealed samples were determined from x-ray reflection measurement using a Bruker X-ray diffractometer, and for the structural characterization of the thin films a Rigaku X-ray diffractometer was used. Magnetic properties were investigated using Quantum Design magnetic property measurement system (MPMS) and magnetotransport properties were studied using Keithley electrometers in conjunction with MPMS.

\section{RESULTS AND DISCUSSION}

Figure 1 shows the $\mathrm{x}$-ray diffraction (XRD) patterns of $\mathrm{Mn}_{55-x} \mathrm{Pt}_{x} \mathrm{Bi}_{45}(x=0$ and 4.5) thin films collected at room

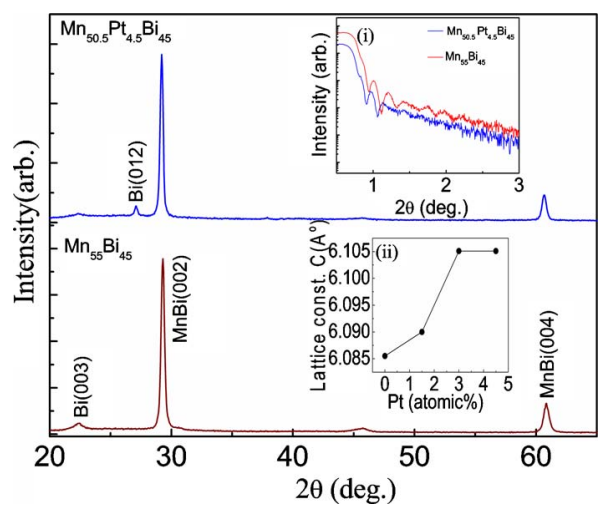

FIG. 1. (Color online) XRD patterns of $\mathrm{Mn}_{55-x} \mathrm{Pt}_{x} \mathrm{Bi}_{45}$ thin films indexed to hexagonal NiAs-type structure. Inset (i) shows the x-ray reflectivity oscillations for samples with 0 at. \% and 4.5 at. \% platinum and inset (ii) shows the change in the c-axis lattice constant with platinum concentration. 


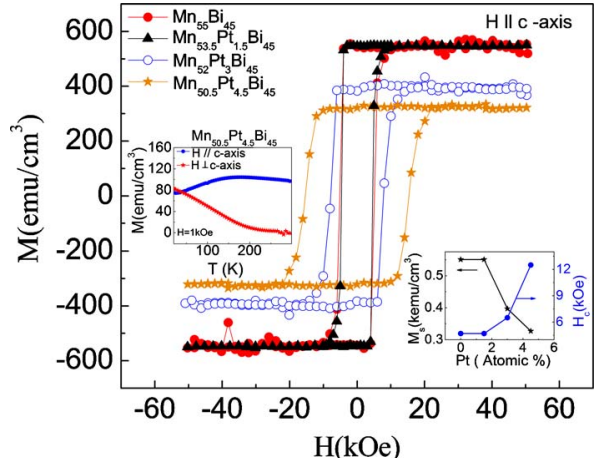

FIG. 2. (Color online) Magnetization as a function of magnetic field for $\mathrm{Mn}_{55-x} \mathrm{Pt}_{x} \mathrm{Bi}_{45}$ thin films. Inset (right): Change in the saturation magnetization $M_{s}$ and the coercivity $H_{c}$ of the samples with Pt concentration. Inset (left): Temperature dependence of the magnetization for $\mathrm{Mn}_{50.5} \mathrm{Pt}_{4.5} \mathrm{Bi}_{45}$ thin film measured in two perpendicular directions.

temperature. The diffraction peaks in the XRD patterns can be indexed to the hexagonal $\mathrm{MnBi}$ crystal structure with space group P63/mmc, although a small trace of unannealed bismuth has been detected. The presence of only the MnBi (002) and (004) reflections indicates that the films are highly $\mathrm{c}$-axis oriented. We have noticed that the intensity of $\mathrm{MnBi}$ (002) peak decreases and $\mathrm{Bi}(012)$ peak increases with the increase in Pt concentration. This indicates that the substitution of Pt into MnBi lattice may cause structural disorder. The estimated values of c-axis lattice parameter from the position of the most intense (002) peak are very close to the value $(c=6.12 \AA)$ reported for the LTP MnBi. This suggests that the samples have adopted the LTP of MnBi. As shown in the inset (ii) of Fig. 1, there is a small increase in $\mathrm{c}$ with the increase in Pt concentration, which is consistent with the incorporation of $\mathrm{Pt}$ in the octahedral site (Mn site) of $\mathrm{MnBi}$ lattice.

Thin film quality can also be assessed from the nature of the top and bottom interfaces of the thin film. Pt substitution has increased the roughness of the interfaces. The faster damping of x-ray reflectivity oscillations in the Pt alloyed samples, as shown in the inset (i) of Fig. 1, is a consequence of the rough interfaces. The change in the surface morphology due to Pt substitution can be clearly seen in the atomic force microscope micrographs as well. The surface of $\mathrm{Mn}_{55} \mathrm{Bi}_{45}$ film is continuous with root-mean-square (rms) roughness of $1.7 \mathrm{~nm}$ over a $4 \times 4 \mu \mathrm{m}^{2}$ scan area and that of $\mathrm{Mn}_{50.5} \mathrm{Pt}_{4.5} \mathrm{Bi}_{45}$ film is not continuous and have increased rms roughness of $2.6 \mathrm{~nm}$ over the same scan area (Figure not shown).

Figure 2 shows the magnetization $\mathrm{M}$ as a function of magnetic field $\mathrm{H}$ for $\mathrm{Mn}_{55-x} \mathrm{Pt}_{x} \mathrm{Bi}_{45}(x=0,1.5,3$, and 4.5) thin films. $\mathrm{M}(\mathrm{H})$ curves were recorded at room temperature with magnetic field perpendicular to the plane of the samples. We have found that the saturation magnetizations $\left(M_{s}\right)$ of $\mathrm{Mn}_{55_{-} x} \mathrm{Pt}_{x} \mathrm{Bi}_{45}$ films decrease monotonically with the increase in Pt concentration but the coercivities $\left(H_{c}\right)$ increase as the $\mathrm{Pt}$ concentration increases. As shown in the right inset of Fig. 2, $M_{s}$ decreases from 551 to $327 \mathrm{emu} / \mathrm{cm}^{3}$ and $H_{c}$ increases from 4.7 to $12.5 \mathrm{kOe}$ as Pt concentration increases from 0 to 4.5 at. \%, although most of the change occurs between 1.5 and 4.5 at. \%. Furthermore, the coercivities of

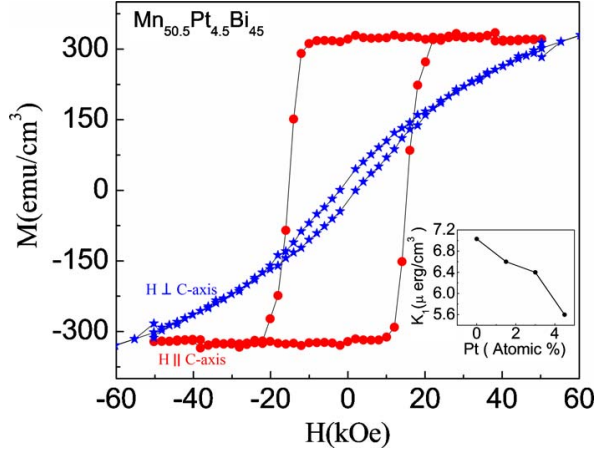

FIG. 3. (Color online) Magnetization as a function of magnetic field for $\mathrm{Mn}_{50.5} \mathrm{Pt}_{4.5} \mathrm{Bi}_{45}$ thin film measured in two perpendicular directions- $H$ parallel to the sample plane $(H \perp \mathrm{c}$-axis) and $H$ perpendicular to the sample plane $\left(H \| \mathrm{c}\right.$-axis). Inset shows the change in anisotropy constant $K_{1}$ with platinum concentration.

all the $\mathrm{Mn}_{5{ }_{5-x}} \mathrm{Pt}_{x} \mathrm{Bi}_{45}$ thin films decrease with the decrease in temperature, indicating that the samples are LTP even after Pt substitution. It has been found that the out-of-plane magnetization measured at $1 \mathrm{kOe}$ of the samples shows a gradual decrease and in-plane magnetization shows a gradual increase as the temperature decreases below $200 \mathrm{~K}$. However, this feature disappears when the $\mathrm{M}(\mathrm{T})$ measurement is done above $10 \mathrm{kOe}$. This is attributed to the gradual deviation in magnetic moment from the c-axis which finally flips into the a-b plane. A representative $\mathrm{M}(\mathrm{T})$ plot for the sample with $4.5 \% \mathrm{Pt}$ is shown in the left inset of Fig. 2.

In order to determine the uniaxial anisotropy constants $K_{1}$ and $K_{2}$, we have recorded $\mathrm{M}(\mathrm{H})$ loops both in the parallel and perpendicular directions. Representative $\mathrm{M}(\mathrm{H})$ loops for $\mathrm{Mn}_{50.5} \mathrm{Pt}_{4.5} \mathrm{Bi}_{45}$ thin film are shown in Fig. 3. The samples maintain their large perpendicular anisotropy but platinum substitution significantly lowers the values of $K_{1}$. We have determined the values of anisotropy constants for these samples using $E=K_{1} \sin ^{2} \theta+K_{2} \sin ^{4} \theta-H M_{s} \sin \theta$ and the Sucksmith-Thompson method, ${ }^{15}$ where $\mathrm{E}$ is the energy density, $K_{1}$ and $K_{2}$ are the anisotropy constants, $M_{s}$ is the saturation magnetization, and $\theta$ is the angle between easy axis and magnetization. As shown in the inset of Fig. 3, $K_{1}$ decreases from $7.03 \times 10^{6} \mathrm{ergs} / \mathrm{cm}^{3}$ for $\mathrm{Mn}_{55} \mathrm{Bi}_{45}$ thin films to $5.60 \times 10^{6} \mathrm{ergs} / \mathrm{cm}^{3}$ for $\mathrm{Mn}_{50.5} \mathrm{Pt}_{4.5} \mathrm{Bi}_{45}$ films.

As explained in the introduction, the phase structure of $\mathrm{MnBi}$ is very complicated, even in the bulk. However, a key feature of the system is the presence of large bipyramidal interstices in the NiAs structure, which can easily be occupied by the Mn atoms. As pointed out by Roberts, ${ }^{16}$ each of these interstitial atoms is coordinated by five $\mathrm{Mn}$ atoms so that a single $\mathrm{Mn}$ atom with antiferromagnetic coupling yields a substantial reduction in the moment. Such a reduction is very likely because $\mathrm{Mn}$ and most $\mathrm{Mn}$ compounds exhibits a pronounced trend toward antiferromagnetism (AFM). AFM is generally favored for half-filled shells because the energy of electron in a filled $3 d \uparrow$ subband is equal to the on-site energy, whereas the antiferromagnetic state can reduce its energy by interatomic hopping. ${ }^{17}$ Ordered $\mathrm{MnBi}$ is an exception, with a atomic structure and a charge state (essentially $\mathrm{Mn}^{3+}$ ) supporting ferromagnetism. In the present system, the Mn-rich regions around the interstices are closer to antifer- 


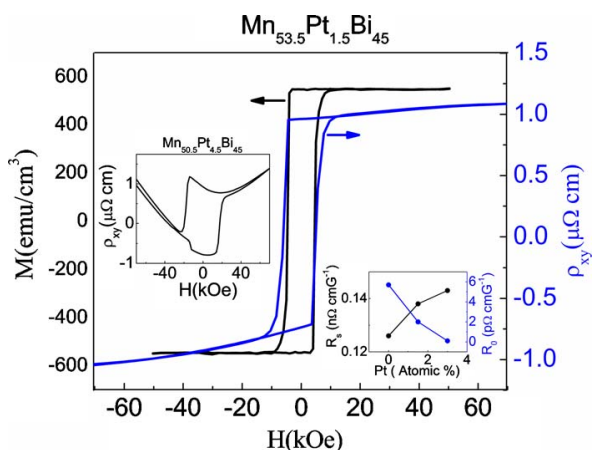

FIG. 4. (Color online) Magnetization $M$ and Hall resistivity $\rho_{x y}$ as a function of magnetic field for $\mathrm{Mn}_{53.5} \mathrm{Pt}_{1.5} \mathrm{Bi}_{45}$ thin film. Inset (right) shows the change in the ordinary Hall coefficient $\left(R_{0}\right)$ and extraordinary Hall coefficient $\left(R_{s}\right)$ with platinum concentration. Inset (left): Hall resistivity as a function of magnetic field for $\mathrm{Mn}_{50.5} \mathrm{Pt}_{4.5} \mathrm{Bi}_{45}$ film.

romagnetic bulk Mn. The excess Mn pushes the system toward antiferromagnetic (or noncollinear) order, and the Pt atoms further increase the interstitial Mn occupancy by displacing the Mn atoms from their regular sites. The effect is further enhanced by the Pt atoms on the regular lattice sites, which further dilute and probably reverse the FM interactions of $\mathrm{MnBi}$.

The coercivity scales as $H_{o}=2 K_{1} / M_{s}$ but it also exhibits a strong dependence on the defect structure ${ }^{18}$ and some dependence on $K_{2}{ }^{19}$ Since $K_{2}$ does not change very much, from $2.5 \times 10^{6} \mathrm{ergs} / \mathrm{cm}^{3}$ for $\mathrm{Mn}_{55} \mathrm{Bi}_{45}$ to 2.06 $\times 10^{6} \mathrm{ergs} / \mathrm{cm}^{3}$ for the sample with $4.5 \% \mathrm{Pt}$, we ignore the effect of $K_{2}$ on the coercivity. The $K_{1}$-only anisotropy field increases from $H_{o}=25.4 \mathrm{kOe}(0 \% \mathrm{Pt})$ to $34.5 \mathrm{kOe}(4.5 \% \mathrm{Pt})$ but this increase is insufficient to explain the observed increase in coercivity. The ratio $H_{c} / H_{o}$ increases from 0.185 for $0 \% \mathrm{Pt}$ to 0.362 for $4.5 \% \mathrm{Pt}$. Both ratios, especially the second one, are large and indicate a very effective pinning mechanism. The increase due to $\mathrm{Pt}$ substitution indicates substantial structural inhomogeneities, which is consistent with our finding that the Pt enhances deviations from the perfect NiAs structures and also produces discontinuities on the film surface. Details of the pinning mechanisms are not known at present but the domain wall energy $\gamma=4\left(A K_{1}\right)^{1 / 2}$ strongly depends on the interatomic exchange $A \sim z J$, so that nanoscale regions with strongly reduced exchange $J$ and/or z are effective pinning centers.

Besides investigating structural and magnetic properties, we have studied the galvanomagnetic properties of $\mathrm{Mn}_{55_{-}} \mathrm{Pt}_{x} \mathrm{Bi}_{45}$ thin films. We have carried out Hall measurement on these samples using the van der Pauw method. The samples exhibit a large extraordinary Hall effect. Hall resistivity $\rho_{x y}(H)$ loops for samples with platinum concentration of $0,1.5$, and 3 at. $\%$ are essentially identical to their $\mathrm{M}(\mathrm{H})$ hysteresis loops, as shown in Fig. 4. However, $\rho_{x y}(H)$ for the sample with 4.5 at. \% Pt shows a quadratic dependence on magnetic field, indicating that the ordinary magnetoresistance starts dominating as the Pt concentration increases, as shown in the left inset in Fig. 4. We have determined the normal and anomalous Hall coefficients $R_{o}$ and $R_{S}$ by fitting $\rho_{x y}=R_{o} B+4 \pi R_{s} M_{s}$ to the high field $\rho_{x y}(H)$ data, where $\mathrm{B}$ is the magnetic induction and $M_{s}$ is the saturation magnetization. ${ }^{20}$ For all the samples, the $R_{s}$ values are about one order of magnitude larger than $R_{o}$ values, which is consistent with past research. ${ }^{21}$ As shown in the right inset of Fig. $\quad 4, \quad R_{s}$ increases from $1.26 \times 10^{-10}$ to 1.49 $\times 10^{-10} \Omega \mathrm{cm} / \mathrm{G}$ and $R_{o}$ decreases from $5.7 \times 10^{-12}$ to 1.0 $\times 10^{-13} \Omega \mathrm{cm} / \mathrm{G}$ as the Pt concentration increases from 0 to 3 at. \%. We could not precisely determine the values of $R_{o}$ and $R_{s}$ for the sample with $4.5 \%$ Pt due to the quadratic dependence of $\rho_{x y}$ on $\mathrm{H}$. The increase in $R_{s}$ and decrease in $R_{o}$ is consistent with the increase in resistivity of the samples due to Pt substitution. ${ }^{21}$

\section{CONCLUSIONS}

We have investigated the structural, magnetic and magneto-transport properties of $\mathrm{Mn}_{55_{-}} \mathrm{Pt}_{x} \mathrm{Bi}_{45}(x=0,1.5,3$, and 4.5) thin films prepared by e-beam multilayer deposition and annealing. We have found that the magnetic properties of $\mathrm{MnBi}$ are very sensitive to the substitution of a third element such as Pt. Saturation magnetization and coercivity of the samples show a systematic change with the Pt concentration. The substantial decrease in magnetization and increase in the coercivity of the Pt-alloyed samples can be attributed to the defect structure with strong pining centers. The increase in the resistivity of the samples due to platinum substitution causes an increase in the extraordinary Hall coefficient and decrease in the ordinary Hall coefficient.

\section{ACKNOWLEDGMENTS}

We would like to thank S. Jaswal for a discussion of exchange interactions in $\mathrm{MnBi}$ and Tom George, Steve Michalski, and Lanping Yue for their assistance in thin-film deposition and measurements. This work is supported by the NSF MRSEC Grant No. DMR-0820521 (P.K. and R.D.K.), DOE Grant No. DE-FG02-04ER46152 (R.S. and D.J.S.), and NCMN.

${ }^{1}$ Di. Chen, G. N. Otto, and F. M. Schmit, IEEE Trans. Magn. 9, 66 (1973). ${ }^{2}$ G. Q. Di, S. Iwata, S. Tsunashima, and S. Uchiyama, J. Magn. Magn. Mater. 104-107, 1023 (1992).

${ }^{3}$ R. R. Heikes, Phys. Rev. B 99, 446 (1955).

${ }^{4}$ X. Guo, X. Chen, Z. Altounian, and J. O. Ström-Olsen, J. Appl. Phys. 73, 6275 (1993).

${ }^{5}$ U. Rüdiger and G. Güntherodt, J. Appl. Phys. 88, 4221 (2000).

${ }^{6}$ T. Chen, J. Appl. Phys. 45, 2358 (1974).

${ }^{7}$ Tu. Chen, IEEE Trans. Magn. 10, 581 (1974)

${ }^{8}$ Di. Chen, J. Appl. Phys. 42, 3625 (1971).

${ }^{9}$ S. S. Jaswal, J. X. Shen, R. D. Kirby, and D. J. Sellmyer, J. Appl. Phys. 75, 6346 (1994)

${ }^{10}$ A. Katsui, J. Appl. Phys. 47, 4663 (1976).

${ }^{11}$ J. X. Shen, R. D. Kirby, and D. J. Sellmyer, J. Appl. Phys. 69, 5984 (1991).

${ }^{12}$ P. R. Bandaru and T. D. Sands, J. Appl. Phys. 86, 1596 (1999).

${ }^{13}$ J. Köhler and J. Kübler, J. Phys.: Condens. Matter 8, 8681 (1996).

${ }^{14}$ U. Rüdiger, P. Fumagalli, T. Roos, B. Holländer, and G. Güntherodt, IEEE Trans. Magn. 33, 3241 (1997)

${ }^{15}$ R. Skomski and J. M. D. Coey, Permanent Magnetism (IOP, Bristol, 1999)

${ }^{16}$ B. W. Roberts, Phys. Rev. B 104, 607 (1956).

${ }^{17}$ R. Skomski, Simple Models of Magnetism (University Press, Oxford, 2008).

${ }^{18}$ H. Kronmüller, Phys. Status Solidi B 144, 385 (1987).

${ }^{19}$ The nucleation-field coercivity is independent of $K_{2}$ but depending on the real structure, this is not necessarily true for pinning.

${ }^{20}$ H. C. Yang, L. M. Wang, and H. E. Horng, Phys. Rev. B 64, 174415 (2001).

${ }^{21}$ M. A. Angadi and V. Thanigaimani, J. Mater. Sci. Lett. 7, 717 (1988). 